\title{
Propagation of Solitary Waves in Warm Magnetized Plasma with Two Temperature Electrons
}

\author{
Nirupama Devi and Latika Kalita \\ Department of Mathematics, Cotton College, Guwahati-1, Assam, INDIA
}

Keywords: ion-acoustic solitary waves, two electron plasma, Sagdeev Potential.

\begin{abstract}
Nonlinear wave structure of ion acoustic disturbances is investigated in magnetized plasma consisting of warm ions and two electron components, namely hot and cold. The basic set of fluid equations for the flow variables is reduced to a single equation known as Sagdeev Potential (SP) equation using non perturbative approach. The properties of solitary wave structures are studied by pseudo potential method, which is valid for arbitrary amplitude. The amplitude of the solitary waves and the depth of the potential well are found to decrease with the increase of the direction-cosine of the wave propagation. The ranges of temperature ratios (ion to electrons) for the existence of solitary waves and their effects on the plasma medium are studied in detail and presented graphically for different sets of plasma parameters.
\end{abstract}

\section{Introduction}

The nonlinear propagation of electrostatic (e.g. ion acoustic) excitations in magnetized plasmas has received considerable attention in the last few decades as witnessed by the increasing number of publication [1-7]. Such nonlinear excitations form specific structures of solitary ion acoustic waves in space regions of the Earth as well as in laboratories. Solitary waves are a typical form of nonlinear waves in a medium developed due to the effect of nonlinearity and dispersion. These waves are localized and are called as solitons, which can trap particles in plasma medium and convict them over large distances. Therefore the solitons can contribute to the transportation of energy related with movements of anomalous particles from one region to another in laboratories, astrophysical and space related plasmas.

In most of the works done the plasma models are seen to be considered as simple ion, electron compositions. Models related to multispecies plasma/complex plasma are also getting importance in such studies [8,9]. But many often reports from spacecraft missions (for example- the FAST, Viking, GEOTALL, POLAR) have proved co-existence of two temperature electrons (2TE) populations[10-16]. Whatever be the model considered the resulting solitary waves (solitons) are found either by the method of perturbation for small amplitudes or by nonperturbative technique for finite/large amplitudes $[17,18]$. The propagation characteristics of ion acoustic waves have been studied in 2TE plasmas [19-21]. In such plasmas two groups of electrons having different temperatures are present and the electron velocity distribution may be represented by the super position of two Maxwellains for the isothermal electrons [22]. Mahmood and Masood [23] have studied electron-acoustic solitary waves in such situation by using nonperturbative technique.

Numerous reports in regard to ion acoustic (ionic sound) waves in plasmas can be found as path finder [24-31]. Quite a number of authors have studied ion acoustic waves/solitons in plasmas consisting of two species of hot and cold electron populations. It is very important to note that the presence of an ambient magnetic field affecting a plasma medium give rise to different situations. The plasma behavior becomes much more complicated in terms of ion cyclotron frequencies. Most of the ion acoustic waves are studied in a regime of low frequency under the effects of magnetic field leading to the introduction of wave modes in a regime of mixed wave frequencies.

We consider warm magnetized plasma model containing two temperature electron populations and see the propagation characteristic of ion acoustic solitons. In such plasma the occurrence of compressive/rarefactive solitons is questioned for sub-subsonic limits. In deriving the ideal evolution equations the pseudopotential method has been applied for a quasi-neutral condition for the plasma. Such nonperturbative works are yet be done for magnetized plsma, as it is seen during 
the literature survey. The paper is organized as follows: In Sec. II, we present the basic equations representing our plasma model. Section III shows the derivation of the Sagdeev potential equation for studying arbitrary amplitude solitary waves. Computational results of different parametric ranges for the existence of nonlinear structures are discussed in Sec. IV. Finally, we summarize our overall result in Sec. V.

\section{Basic Equations}

To study the nonlinear waves we have considered the basic equations governing a plasma contaminated with the warm ions and two temperature electron distributions immersed in an uniform external magnetic field $\mathrm{B}_{0}=\mathrm{B}_{0} \mathrm{z}^{\wedge}$ directed along $\mathrm{z}$ - axis. The electron distributions are considered asin ref. [22]. The equations of motion for warm ions in (x-z) plane are

$$
\begin{aligned}
& \frac{\partial n_{i}}{\partial t}+\frac{\partial}{\partial x}\left(n_{i} v_{x}\right)+\frac{\partial}{\partial z}\left(n_{i} v_{z}\right)=0 \\
& \frac{\partial v_{x}}{\partial t}+\left(v_{x} \frac{\partial}{\partial x}+v_{z} \frac{\partial}{\partial z}\right) v_{x}=-\frac{\partial \phi}{\partial x}+v_{y}-\frac{\alpha}{n_{i}} \frac{\partial n_{i}}{\partial x} \\
& \frac{\partial v_{y}}{\partial t}+\left(v_{x} \frac{\partial}{\partial x}+v_{z} \frac{\partial}{\partial z}\right) v_{y}=v_{x} \\
& \frac{\partial v_{z}}{\partial t}+\left(v_{x} \frac{\partial}{\partial x}+v_{z} \frac{\partial}{\partial z}\right) v_{z}=-\frac{\partial \phi}{\partial z}-\frac{\alpha}{n_{i}} \frac{\partial n_{i}}{\partial z} \\
& n_{e}=n_{h}+n_{c}
\end{aligned}
$$

We have normalized the ion, electron densities $n_{i}, n_{e}$ by the equilibrium plasma density $n_{0}$, time (t) by the inverse of the ion gyrofrequency $\Omega_{i}$, velocity by the ion sound speed $C_{s}\left[=\left(T_{e f f} / m_{i}\right)^{1 / 2}\right]$, space by the ion gyro radius $\rho_{s}\left[=C_{s} / \Omega_{i}\right], \Omega_{i}=e B_{0} / m_{i}$ is the ion gyrofrequency, potentials $\phi$ by $T_{\text {eff }} / e$, magnetic field by $B_{0}$. Temperature is normalized to effective temperature $T_{\text {eff }}=T_{h} T_{c} /\left(n_{0 h} T_{h}+n_{0 c} T_{c}\right)$. Here, $\alpha=T_{i} / T_{\text {eff }}, \beta=T_{c} / T_{h}, \mu=n_{c 0} / n_{0}, v=n_{h 0} / n_{0}$.

\section{Derivation of the sagdeev potential (SP) equation}

In order to obtain a traveling wave solution, we consider a stationary wave in the moving frame defined by $\xi=x k_{x}+z k_{z}-M_{t}$, where $M=V / V_{A}$ is the phase velocity of the wave in the unit of the velocity $V_{A}$ (or the Mach number) and $k_{x}^{2}+k_{z}^{2}=1, k_{x}$ and $k_{z}$ are direction cosines. So that

$$
\frac{\partial}{\partial x} \rightarrow k_{x} \frac{\partial}{\partial \xi}, \quad \frac{\partial}{\partial z} \rightarrow k_{z} \frac{\partial}{\partial \xi}, \quad \frac{\partial}{\partial t} \rightarrow-M \frac{\partial}{\partial \xi}
$$

Using Eq. (8), above equations can be reduced to a set of ordinary differential equations in the following form 


$$
\begin{aligned}
& k_{x} v_{x}+k_{z} v_{z}=M\left(1-\frac{1}{n_{i}}\right) \\
& \frac{M}{n_{i}} \frac{\partial v_{x}}{\partial \xi}=k_{x} \frac{\partial \phi}{\partial \xi}-v_{y}+\frac{\alpha}{n_{i}} k_{x} \frac{\partial n_{i}}{\partial \xi} \\
& \frac{M}{n_{i}} \frac{\partial v_{y}}{\partial \xi}=v_{x} \\
& \frac{M}{n_{i}} \frac{\partial v_{z}}{\partial \xi}=k_{z} \frac{\partial \phi}{\partial \xi}+\frac{\alpha}{n_{i}} k_{z} \frac{\partial n_{i}}{\partial \xi} \\
& n_{e}=n_{h}+n_{c}
\end{aligned}
$$

Now differentiating (9) with respect to $\xi$ we get,

$$
\begin{aligned}
& k_{x} \frac{d v_{x}}{d \xi}+k_{z} \frac{d v_{z}}{d \xi}=\frac{M}{n_{i}^{2}} \frac{d n_{i}}{d \xi} \\
& \text { gether with (14) we get }
\end{aligned}
$$$$
\frac{M^{2}}{n_{i}^{3}} \frac{d n_{i}}{d \xi}=\frac{d \phi}{d \xi}+\frac{\alpha}{n_{i}} \frac{d n_{i}}{d \xi}-k_{x} v_{y}
$$

Now differentiating Eq. (15) with respect to $\xi$ and using equations (9), (11) we get,

$$
\frac{d}{d \xi}\left[\left(\frac{M^{2}}{n_{i}^{3}}-\frac{\alpha}{n_{i}}\right) \frac{d n_{i}}{d \xi}\right]=\frac{d^{2} \phi}{d \xi^{2}}-\frac{n_{i}}{M}\left(M-\frac{M}{n_{i}}-k_{z} v_{z}\right)
$$

Taking logarithm, equation (6) can be written as,

$$
\begin{aligned}
& \log \left(n_{h}\right)=\log \left(v \exp \frac{\beta \phi}{\mu+v \beta}\right), \Rightarrow \log \left(n_{h}\right)=\log v+\frac{\beta \phi}{\mu+v \beta} \\
& \qquad \frac{d n_{h}}{d \xi}=\frac{n_{h} \beta}{\mu+v \beta} \frac{d \phi}{d \xi}
\end{aligned}
$$

Similarly from equation (7) we get $\frac{d n_{c}}{d \xi}=\frac{n_{c}}{\mu+v \beta} \frac{d \phi}{d \xi}$

Differentiating equation (13) with respect to $\xi$ and using equations (17) and (18) we get,

$$
\frac{d n_{e}}{d \xi}=\frac{1}{\mu+v \beta}\left[n_{h} \beta+n_{c}\right] \frac{d \phi}{d \xi}
$$

Combination of (6) and (7) gives $n_{h}=\frac{v}{\mu^{\beta}} n_{c}^{\beta}$

Therefore, Eq. (13) becomes

$$
n_{e}=\frac{v}{\mu^{\beta}} n_{c}^{\beta}+n_{c}
$$

Differentiating equation (21) with respect to $\xi$ we get

$$
\frac{d n_{e}}{d \xi}=\left(1+\frac{v \beta}{\mu^{\beta}} n_{c}^{\beta-1}\right) \frac{d n_{c}}{d \xi}
$$


Using (20) in (19) we get,

$\frac{d n_{e}}{d \xi}=\frac{1}{\mu+v \beta}\left(\frac{v}{\mu^{\beta}} n_{c}^{\beta} \beta+n_{c}\right) \frac{d \phi}{d \xi}$

$\Rightarrow \frac{d \phi}{d \xi}=\frac{\mu+v \beta}{n_{c}} \frac{1}{\left(1+\frac{v \beta}{\mu^{\beta}} n_{c}^{\beta-1}\right)} \frac{d n_{e}}{d \xi}$

Using (22) in (23) we get, $\frac{d \phi}{d \xi}=\frac{\mu+v \beta}{n_{c}} \frac{d n_{c}}{d \xi}$

From Eq. (12), integrating with respect to $\xi$ and using boundary condition $v_{z}=0$ at $n_{c}=1$ as $\xi \rightarrow \infty$ and using charge neutrality condition, $n_{i}=n_{e}$ we get

$$
v_{z}=\frac{k_{z}}{M}\left[(\mu+v \beta)\left\{\left(n_{c}+\frac{v}{\beta \mu^{\beta}} n_{c}^{\beta}\right)-\left(1+\frac{v}{\beta \mu^{\beta}}\right)\right\}+\alpha\left\{\left(n_{c}+\frac{v}{\mu^{\beta}} n_{c}^{\beta}\right)-\left(1+\frac{v}{\mu^{\beta}}\right)\right\}\right]
$$

Simplification of equation (16) gives rise to an equation of the form

$$
\begin{aligned}
& \frac{d}{d \xi}\left(\left(\left\{\frac{M^{2}}{\left(n_{c}+\frac{v}{\mu^{\beta}} n_{c}^{\beta}\right)^{3}}-\frac{\alpha}{\left(n_{c}+\frac{v}{\mu^{\beta}} n_{c}^{\beta}\right)}\right\}\left(1+\frac{v}{\mu^{\beta}} \beta n_{c}^{\beta-1}\right)-\frac{\mu+v \beta}{n_{c}}\right) \frac{d n_{c}}{d \xi}\right)=1-\left(n_{c}+\frac{v}{\mu^{\beta}} n_{c}^{\beta}\right) \\
& +\frac{n_{c} k_{z}^{2}}{M^{2}}(\mu+v \beta)\left(n_{c}+\frac{v}{\mu^{\beta}} n_{c}^{\beta}\right)\left(1+\frac{v}{\beta \mu^{\beta}} n_{c}^{\beta-1}\right)-\frac{k_{z}^{2}}{M^{2}}(\mu+v \beta)\left(1+\frac{v}{\beta \mu^{\beta}}\right)\left(n_{c}+\frac{v}{\mu^{\beta}} n_{c}^{\beta}\right) \\
& +\frac{\alpha k_{z}^{2}}{M^{2}}\left(n_{c}+\frac{v}{\mu^{\beta}} n_{c}^{\beta}\right)^{2}-\frac{\alpha k_{z}^{2}}{M^{2}}\left(1+\frac{v}{\mu^{\beta}}\right)\left(n_{c}+\frac{v}{\mu^{\beta}} n_{c}^{\beta}\right)
\end{aligned}
$$

Multiplying both sides of equation (26) by the terms inside the parenthesis and after some rather lengthy but straightforward algebra we obtain an equation of the form

$$
\frac{1}{2}\left(\frac{d n_{c}}{d \xi}\right)^{2}+K\left(n_{c}, \alpha, \beta, \mu, v, M, k_{z}\right)=0
$$

The boundary condition used in deriving equation (27) is $\frac{\partial n_{c}}{\partial \xi}=0, n_{c}=1$ at $\xi= \pm \infty$. Equation (27) can be interpreted as an energy integral of an oscillatory particle of an unit mass with velocity $\frac{d n_{c}}{d \xi}$ and position $\mathrm{n}_{\mathrm{c}}$ in a potential well $K\left(n_{c}\right)$. That is the above equation can be considered as a motion of a particle whose pseudoposition is $\mathrm{n}_{\mathrm{c}}$ at pseudotime $\xi$ with pseudovelocity $\frac{d n_{c}}{d \xi}$ in a pseudopotential well $K\left(n_{c}\right)$. That is why Sagdeev's potential is called pseudopotential and the Sagdeev Potential is given by 


$$
\begin{aligned}
& K\left(n_{c}, \alpha, \beta, \mu, v, M, k_{z}\right)=A\left[\frac{M^{2}}{2}\left\{\frac{1}{\left(n_{c}+\frac{v}{\mu^{\beta}} n_{c}^{\beta}\right)^{2}}-\frac{1}{\left(1+\frac{v}{\mu^{\beta}}\right)^{2}}\right\}\right. \\
& +\left(1-k_{z}^{2}\right) \alpha\left\{\log \left(n_{c}+\frac{v}{\mu^{\beta}} n_{c}^{\beta}\right)-\log \left(1+\frac{v}{\mu^{\beta}}\right)\right\}+\left\{(\mu+v \beta)\left(1-k_{z}^{2}\right)\right\} \log n_{c} \\
& -\left\{\frac{1}{\left(n_{c}+\frac{v}{\mu^{\beta}} n_{c}^{\beta}\right)}-\frac{1}{\left(1+\frac{v}{\mu^{\beta}}\right)}\right\}\left[M^{2}+k_{z}^{2}\left\{(\mu+v \beta)\left(1+\frac{v}{\beta \mu^{\beta}}\right)+\alpha\left(1+\frac{v}{\mu^{\beta}}\right)\right\}\right] \\
& -\left\{\left(n_{c}+\frac{v}{\mu^{\beta}} n_{c}^{\beta}\right)-\left(1+\frac{v}{\mu^{\beta}}\right)\right]\left[\frac{k_{z}^{2}}{M^{2}}(\mu+v \beta)\left\{(\mu+v \beta)\left(1+\frac{v}{\beta \mu^{\beta}}\right)+\alpha\left(1+\frac{v}{\mu^{\beta}}\right)+\frac{M^{2}}{k_{z}^{2}}\right\}\right] \\
& +k_{z}^{2}(\mu+v \beta)\left[\frac{\left(n_{c}+\frac{v}{\beta \mu^{\beta}} n_{c}^{\beta}\right)}{n_{c}\left(1+\frac{v}{\mu^{\beta}} n_{c}^{\beta-1}\right)}-\frac{\left(1+\frac{v}{\beta \mu^{\beta}}\right)}{\left(1+\frac{v}{\mu^{\beta}}\right)}\right]+\frac{k_{z}^{2} \alpha^{2}}{2 M^{2}}\left\{\left(n_{c}+\frac{v}{\mu^{\beta}} n_{c}^{\beta}\right)^{2}-\left(1+\frac{v}{\mu^{\beta}}\right)^{2}\right\} \\
& +\frac{k_{z}^{2} \alpha}{M^{2}}(\mu+v \beta)\left[\left(n_{c}^{2}-1\right)+\left(\frac{v}{\mu^{\beta}}\right)^{2} \frac{\left(n_{c}^{2 \beta}-1\right)}{\beta}+\left(2 v+v \beta+\frac{v}{\beta}\right) \frac{1}{\mu^{\beta}} \frac{\left(n_{c}^{\beta+1}-1\right)}{\beta+1}\right] \\
& \left.+\frac{k_{z}^{2}}{M^{2}}(\mu+v \beta)^{2}\left[\frac{\left(n_{c}^{2}-1\right)}{2}+\left(v+\frac{v}{\beta}\right) \frac{\left(n_{c}^{\beta+1}-1\right)}{\mu^{\beta}(\beta+1)}+\frac{v^{2}}{2 \mu^{2 \beta}} \frac{\left(n_{c}^{2 \beta}-1\right)}{\beta^{2}}\right]\right] \\
& \text { where, } A=\left[\left\{\frac{M^{2}}{\left(n_{c}+\frac{v}{\mu^{\beta}} n_{c}^{\beta}\right)^{3}}-\frac{\alpha}{\left(n_{c}+\frac{v}{\mu^{\beta}} n_{c}^{\beta}\right)}\right\}\left(1+\frac{v \beta}{\mu^{\beta}} n_{c}^{\beta-1}\right)-\frac{\mu+v \beta}{n_{c}}\right]^{-2}
\end{aligned}
$$

The conditions for existence of solitary waves are as follows [Writing $K\left(n_{c}, \alpha, \beta, \mu, v, M, k_{z}\right)$ as $\left.K\left(n_{c}\right)\right]$,

(i) $K\left(n_{c}\right)<0$ between $\mathrm{n}_{\mathrm{c}}=1$ and $\mathrm{n}_{\mathrm{c}}=\mathrm{N}$, so that $\mathrm{dnc} / \mathrm{d} \xi$ is real [Eq. (27)], here $\mathrm{N}$ gives the amplitude of the solitary wave. $\mathrm{N}$ can be both greater than 1 and less than 1 . In the former case we have compressive solitary wave and in the latter case rarefactive solitary waves.

(ii) $K\left(n_{c}\right)$ must be a maximum at $n_{c}=1$ which means, $\left.\frac{\partial K\left(n_{c}\right)}{\partial n_{c}}\right|_{n_{c}=1}=0$ and $\left.\frac{\partial K^{2}\left(n_{c}\right)}{\partial n_{c}{ }^{2}}\right|_{n_{c}=1}<0$

(iii) $K\left(n_{c}\right)$ should cross the ' $n_{c}$ ' axis from below near $n_{c}=N$; and $K\left(n_{c}\right)>0$ for $n_{c}>N$. 


\section{Results And Discussions}

In our present plasma model with warm ion and two electron temperature, we have investigated the formation of arbitrary amplitude compressive solitary waves. The existence of large amplitude solitary waves can be determined by plotting $K\left(n_{\mathcal{C}}\right)$ against density $n_{\mathcal{C}}$ for different values of plasma parameters. To study the effect of each individual parameter on the formation of nonlinear structures, we would vary one parameter at a time and keep other parameters fixed.

In figure1 the Sagdeev Potential $K\left(n_{\mathcal{C}}\right)$ versus density $n_{\mathcal{C}}$ is plotted for different plasma parameters by using equation (28). It is observed that the amplitude of solitary wave increases with decrease of $\mu\left(=n_{c} 0 / n_{0}\right)$ for $M=0.1, v=0.09, \beta=0.9, \alpha=0.02, k_{z}=0.009$ and three different values of $\mu=95$ (solid line), 100 (dotted line), 105 (dashed line). We have varied $\mu \leq 85.3$ and noticed that for lower values of $\mu=85.3$ solitary waves do not exist. Compressive solitary waves are found to exist for $M$ less than 1 . Next we discussed the effect of parameter $\beta\left(=T_{C} / T_{h}\right)$, on nonlinear wave structures. To observed that, we kept all other parameters fixed and varied $\beta$ within the parametric range. The amplitudes and depths of solitary waves are found to be increasing as $\beta$ increseses, which is depicted in figure 2 for $M=0.1, v=0.09, \mu=100, \alpha=0.02, k_{z}=0.009$ and different values of $\beta=0.3$ (solid line), 0.38 (dotted line), 0.45 (dashed line) and 0.56 (dotted line). The curve for $\beta=0.3$ (solid line) does not represent any of those nonlinear wave structures. Solitary wave exists for upper values of $\beta=0.372$. Here we have observed that for lower values of $\beta$ over the range 0.01- 0.09, 0.001-0.009, solitary wave does not exist. Figure 3 shows the existence of solitary waves for different values of $k_{z}=0.0079$ (solid line), 0.0086 (dotted line), 0.0089 (dashed line), 0.0094 (dotted line). It is seen that amplitude increases as $k_{z}$ decreases. From our observation, if $k_{z}<0.008498, K\left(n_{c}\right)$ does not satisfy the existence of solitary waves and hence soliton solution does not exist. So, it is observed that amplitude of the nonlinear wave structures depends on the external magnetic field $k_{z}$. The temperature ratio $\alpha\left(=T_{i} / T_{\text {eff }}\right)$ also plays important role in the formation of the non-linear structures which is depicted in Fig. 4 for fixed $M=0.1, v=0.09$, $\mu=100, \beta=0.9, k_{z}=0.009$ and different values of $\alpha=0.00192$ (solid line), 0.058 (dotted line) and 0.2 (dashed line). We have notice that the amplitude increases as $\alpha$ decreases. Solitary wave exists for lower values of $\alpha \leq 0.4$, when $\alpha>0.5$ solitary wave does not exists. The amplitudes and depths are found to be increasing as $M$ (Mach number) increases, which is plotted in figure 5 for $v=0.09$, $\mu=100, \beta=0.9, \alpha=0.02, k_{z}=0.009$ and three different values of $M=0.092$ (dashed line), 0.096 (dotted line) and 0.098 (solid line).

Here we have varied $M$ over a range $0.01-0.091$, in that region solitary wave does not exist. When $M \geq 0.092$ the soliton solution exists. Figure 6 shows that Sagdeev potential $K\left(n_{\mathcal{C}}\right)$ vs density $n_{\mathcal{C}}$ is plotted for $M=0.1, \mu=100, \beta=0.9, k_{z}=0.009, \alpha=0.02$ and $v=0.005$ (solid line).

\section{Conclusion}

The set of basic equations governing the warm ion dynamics, Boltzmannian distribution for two electron temperature together with continuity equation have been reduced to a single equation known as Sagdeev Potential (SP) equation. An exact analytical expression for the energy integral is obtained and analyzed numerically through which compressive solitary waves of arbitrary amplitude are found to exist for different regions of various plasma parameters. Effects of different parameters on the amplitudes on the nonlinear wave structures are investigated. Our present theoretical studies could be of interest and it is expected to explain some of the recent spacecraft observations (e. g. Freja, Viking, GEOTAIL and POLAR etc.) in space plasma where two temperature electrons distribution are present.

\section{Acknowledgements}

The authors are grateful to the Head of the Department of Mathematics, Cotton College, Guwahati, Assam for encouraging research works. 


\section{References}

[1] Shukla P. K. and Yu M. Y., J. Math. Phys., 19(12), 1978, pp. 2506-08. [2] Yu, Shukla P. K., Bujarbarua, Phys. Fluids, 23, 1980, pp. 2146-2147.

[3] Kalita B. C., Kalita M. and Chutia J., J. Phy. A. Math. Gen. 19, 1980, pp. 3559-3563. [4] Song

L. T., Lee L. C. and Huang L., Phys. Fluids, 31(6), 1988, pp. 1549.

[5] Shivamoggi B. K., J. Plasma Physics, Vol. 41 (Part I), 1989, pp. 83-88.

[6] Kalita B. C. and Bhatta R. P., Phys. Plasmas, Vol. I, No. 6, 1994, pp. 2172.

[7] Akbari-Moghanjoughi M., Phys. Plasmas, 18, 2011, pp. 032103.

[8] Nakamura Y., J. Plasma Physics, Vol. 38, Part 3, 1987, pp. 416-471.

[9] Gogoi R., Devi N., Das G. C., Indian Journal of Pure \& Applied Physics, Vol. 46, 2008, pp. 621-628.

[10] Temerin M., Cerny K., Lotko W. and Mozer F. S., Phys. Rev. Lett., 48, 1982, pp. 1175.

[11] Boström R., Phys. Rev. Lett., 61, 1988, pp. 82.

[12] Ergun R. E. et al., Geophys Res. Lett., 25, 1998, pp. 2061 ; Delory G. T., Ergun R. E., Carlson C. W., Muschietti L., Chaston C. C., Peria W., McFadden J. P. and Strangeway R., Geophys Res. Lett., 25, 1998, pp. 2069.

[13] McFadden J. P., Carlson C. W., Ergun R. E., Mozer F. S., Muschietti Roht I. and Moebius E., Geophys Res. Lett., 108, 2003, pp. 8018.

[14] Matsumoto H. et al. Geophys. Res. Lett., 21, 1994, pp. 2915.

[15] Franz J. R. et al. Ibid, 25, 1998, pp. 1277.

[16] Cattell C.A. et al. Ibid, 26, 1999, pp. 425.

[17] Washimi H. \& Tanuity T., Phys Rev Lett, 17, 1966, pp. 996.

[18]. Sagdeev R. Z, Reviews of Plasma Physics (Consultants Bureau, New York), vol 4, 1966, pp. 23.

[19] Jones W. D., Lee A., Gleman S. M., and Doucet H. J., Phys. Rev. Lett. 35, 1975, pp. 1349.

[20] Goswami B. N. and Buti B., Phys. Lett. 57A, 1976, pp. 149.

[21] Karmakar B., Das G. C., and Singh Kh. I., Int. J. Theor. Phys. 29, 1990, pp.1099.

[22] Tagare S. G., Phys. Plasmas, Vol. 7, 2000, No. 3.

[23] Mahmood S. and Masood W., Phys. Plasmas, 15, 2008, 122302,

[24] Tonks L. and Langmuir I., Phys. Rev. 33, 1929, pp. 195.

[25] Alexeff I., Neidigh R. V., Phys. Rev. Lett., 7, 1961, pp. 223.

[26] Widner M, Alexeff I., Jones W. D., Lonngren K. E., Phys. Fluids, 13, 1970, pp. 2532.

[27] Ikezi H., Kiwamoto Y., Lonngren K. E., Phys. Plasmas, 15, 1973, pp. 1141.

[28] Longren K .E., Khazei M., Gable E. F., Bulson J. M., Phys. Plasmas, 24, 1982, pp. 1483.

[29] Cross R. C., Phys. Plasmas, 25, 1983, pp. 1377.

[30] Singh A. K., Sexena Y. C., J. Plasma Phys., 58, 1996, pp. 623.

[31] Li Y. F., Ma J. X., Li Y. R., Xiao D. L., Lonngren K. E., Phys. Lett., 358A, 2006, pp. 297. 